\title{
Construction of filter vectors for the
}

\section{information-efficient spectral imaging sensor}

\author{
B. R. Stallard and S. M. Gentry \\ Sandia National Laboratories, Albuquerque, NM 87185-0980
}

RECEIVED

OCT 021998

OSTI

\begin{abstract}
The information-efficient spectral imaging sensor (ISIS) seeks to improve system performance by processing hyperspectral information in the optical hardware. Its output may be a gray scale image in which one attempts to maximize the contrast between a given target and the background. Alternatively, its output may be a small number of images, rather than a full data cube, that retain the essential information required in the application. The principal advantage of ISIS is that it offers the discrimination of hyperspectral imaging while achieving the signal-to-noise ratio of multispectral imaging. The paper focuses on construction of the filter vectors that are needed to program ISIS. The instrument produces an image which is essentially a dot product of the scene and the filter vector. Both single vector and multiple vector approaches are considered. Also, we discuss some subtle points related to optimizing the filter vectors.
\end{abstract}

KEYWORDS: imaging spectrometry, linear discriminant analysis, optical data compression

\section{INTRODUCTION}

Remote sensing by satellite is increasingly important to national governments for treaty verification, battlefield monitoring, and other activities. In addition, civilian oriented applications are increasing in areas such as geology, meteorology, ecology, forestry, and agriculture. Spectral imaging sensors, an important subclass of satellite-borne sensors, have been shown to provide information far superior to that of conventional panchromatic images in many of these applications. However, spectral imaging adds at least two challenges to the already difficult task of viewing the earth from a distance of hundreds of kilometers. First, with numerous spectral channels, the signal-to-noise ratio (SNR) may be poor in any one channel. Second, the data rate of a hyperspectral imaging sensor (HSI), which may be $10 \mathrm{Mbytes} / \mathrm{sec}$, or more, stresses the limits of the electronic systems, including the onboard data storage, the downlink bandwidth, and the earthbound image analysis system. This report describes a new concept, the information-efficient spectral imaging sensor (ISIS), which addresses these two problems while offering the promise of nearly real-time identification of targets.

We begin this paper with a brief discussion of the principles of ISIS. This subject is covered in more depth in a companion paper at this conference and two internal reports from Sandia National Laboratories. ${ }^{1-3}$ Then we present some of the issues related to calculating the filter vectors that are to be programmed into ISIS. With much work yet to be accomplished, this paper is essentially a progress report.

\section{PRINCIPLES OF ISIS}

Figure 1 is a schematic diagram of one embodiment of the ISIS instrument, intended for pushbroom imaging. In the ISIS instrument the dispersed light passes through a spatial light modulator, which transmits varying amounts of each wavelength, and then is recombined by a second disperser acting in a negative mode. The filtered light is focused onto a linear array detector at the exit slit which is oriented so that the array is parallel to the slit. Two arms are shown which allows images from two separate filter functions to be collected simultaneously. Note that figure 1 can be converted into a diagram of a conventional HSI by discarding one of the arms and replacing the spatial light modulator with a two-dimensional detector array. In ISIS, the fact that the light is recombined before it is detected provides a multiplex advantage thereby increasing the SNR. The exact SNR enhancement is a complex function of the specific filter vector, but, in a model calculation, is seen to be about $5-10 \mathrm{x}$ that of a conventional HSI. ${ }^{3}$ 


\section{DISCLAIMER}

This report was prepared as an account of work sponsored by an agency of the United States Government. Neither the United States Government nor any agency thereof, nor any of their employees, makes any warranty, express or implied, or assumes any legal liability or responsibility for the accuracy, completeness, or usefulness of any information, apparatus, product, or process disclosed, or represents that its use would not infringe privately owned rights. Reference herein to any specific commercial product, process, or service by trade name, trademark, manufacturer, or otherwise does not necessarily constitute or imply its endorsement, recommendation, or favoring by the United States Government or any agency thereof. The views and opinions of authors expressed herein do not necessarily state or reflect those of the United States Government or any agency thereof. 


\section{DISCLAIMER}

Portions of this document may be illegible in electronic image products. Images are produced from the best available original document. 
Reference 3 compares the SNR of an HSI and ISIS instrument after the data analysis is complete. Therefore, the fact that the data analysis improves the effective SNR of the HSI is not ignored. With ISIS, the best predicted SNR is found when the design takes advantage of both dimensions in the plane of the exit slit. In other words, by removing both slits, and placing several linear array detectors at the focal plane one essentially multiplies the number of ISIS instruments contributing to the final signal averaged result. We are testing the use of a time delay and integrate focal plane in ISIS in order to capitalize on this potential. ${ }^{3}$

Spectroscopists will recognize figure 1 as essentially a Hadamard spectrometer. ${ }^{4}$ ISIS, however, has several features that make it much more that a simple Hadamard spectrometer. First, the slit image is retained from entrance to exit so that pushbroom imaging is possible. Some care in the instrumental design is required to produce a high quality image. The specific design will be discussed in a future publication. Another difference is that, in ISIS, there is a programmable filter rather than a set of fixed Hadamard masks. In our first two instruments the filters have been liquid crystal spatial light modulators, although other types of spatial light modulators may be attractive. ${ }^{1}$ Also, the filter is gray scale rather than merely binary as in the Hadamard approach. Finally, we have given ISIS two channels so that both the positive and negative component of a calculated vector can be collected simultaneaously. ${ }^{1}$ Numerous variations on the ISIS theme are possible. The basic description in the present work pertains to the two instruments that have been constructed and operated at Sandia.

\section{SINGLE FILTER VECTOR RESULT}

Simply put, the function of ISIS is to produce a dot product of the image with a filter vector that is programmed into the instrument. Figure 2 shows a photograph of a laboratory scene and an ISIS image of that scene, where a single vector was chosen to discriminate the artificial ivy from both the real ivy and the cardboard background. The image has been thresholded so that each pixel is either 0 or 1 . This image was collected with the Phase I instrument in a laboratory setting using only the wavelength range 500 to $750 \mathrm{~nm}$. Images from the Phase II instrument which operates over the whole Si detector range of 450 to $1050 \mathrm{~nm}$ will be included in future publications. Also, results from field tests, rather than merely laboratory tests, will be forthcoming. Nevertheless, this laboratory results gives us a basis to discuss the filter vector construction, which is the focus of this paper.

Figure 3 shows the mean spectra for the three materials in this test case. Figure 4 is a scatter plot of the pixels from the scene of figure 2 imaged with a conventional HSI (not shown) equipped with a Si detector. In figure 4 , where the axes are the first three principle components (PC), the pixels of the three materials form distinct clusters. To obtain the ISIS image in figure 2 we first calculated a filter vector (see figure 5) based on the HSI data. The objective is to find an axis on which to project each image pixel so that a histogram of the scores will optimally separate the target material from the other two. The mathematical computation will be discussed below. Figure 6 shows the histogram of the scores and the threshold used to produce the ISIS image of figure 2.

The filter vector, $D$, of figure 5 is found by linear discriminant analysis ${ }^{5}$ according to the following equation:

$$
D=\left(\frac{\Sigma_{1}+\Sigma_{2}}{2}\right)^{-1}\left(M_{2}-M_{1}\right)
$$

where $\Sigma$ is the covariance matrix of class one and two as designated by the subscript, and $M$ is the mean spectrum of the classes. The exponent indicates a matrix inversion. Note that in figure 2 the first target class is the artificial ivy while the second class is a combination of the real ivy and cardboard background. The filter vector calculated by equation 1 can be scaled by an arbitrary factor. 
It is useful to define a separation metric (SM) based on the normalized separation of the two classes:

$$
S M=\frac{\sqrt{\frac{\sigma_{1}^{2}+\sigma_{2}^{2}}{2}}}{\Delta},
$$

where $\sigma$ is the Gaussian width of each class, in a one dimensional representation such as figure 6 , and delta is the separation of the means of the two classes. Clearly the distributions may not be strictly Gaussian but this still serves as a useful metric as long as one checks that the distributions are not radically deviant from Gaussian.

\section{SPECIAL CONSIDERATIONS}

In this section we discuss two subtleties of applying equation 1 . If one applies equation 1 to the full spectra (in this case, 96 channels, from about 500 to $1000 \mathrm{~nm}$ ) the result is the filter vector labeled 96 in figure 7 . Note that, here, the classes are different from figures 2,5 and 6 . We now consider artificial plant versus real plant with no consideration of the cardboard spectra. The fine structure on the filter vector of figure 7-96 is clearly much greater than in the mean spectra for the classes as recorded in figure 3. We take this as evidence that the filter vector is overfit, which is undesirable for two reasons. First, overfitting makes the filter vector scene specific, thus it is likely to produce an optimistic SM when applied to the training scene and a pessimistic SM when applied to a related scene. We prefer a robust filter vector that can be successfully applied to a variety of similar scenes. Second, the complex vector has a high number of zero crossings which will degrade the SNR of the ISIS instrument due to low throughput as described in reference 3.

Rather than apply equation 1 directly, we first reduce the dimension of the data by converting the spectra to their PC scores. Equation 1 is then applied to the PC scores with the final step being a conversion of the calculated filter vector back into the original 96 dimensional spectral space. But how many PCs should be retained in the calculation? Figure 7 shows how this choice affect the resulting filter vector. A filter vector that has a similar amount of fine structure as the mean spectra is an appropriate choice. Another approach is to consider the eigenvalues computed along with the PCs. For this example, $99.9 \%$ of the variance is captured in the first 5 or so PCs. Still another approach to deciding how many PCs to retain is illustrated in Figure 8. We see how the SM varies with the number of PCs retained. Clearly most of the separation is accomplished with the first 5-10 PCs.

Statisticians have recognized the problem of overfitting in linear discriminant analysis. The truncated PC expansion approach, used here, is a simple solution. An interesting and more sophisticated approach known as penalized discriminant analysis is described in reference 6.

A second subtlety in constructing filter vectors is illustrated in figure 9. The spatial light modulator in the ISIS instrument can take away but not add light. Furthermore, the sensitivity of the instrument falls away at the edges of the detector's spectral range. If we have a high point in the filter at the edge of the sensitivity curve, as in figure 9a, we must suppress the other channels considerably in order to produce the desired filter function. In this case it may be better to ignore the filter function near the edges of the detector sensitivity, i.e., turn off the pixels entirely. This is illustrated in figure $9 \mathrm{~b}$. Now the filter vector can be scaled upward until another point touches the detector sensitivity curve, thereby increasing the throughput. Note that the filter vector in figure 9 is a contrived example and not related to figure 2 . In order to make judgements regarding these edge effects we must have a way to explore the trade space. In figure 10 we plot the SM for the data related to figure 2 as a function of various combinations of excluded spectral channels from both the right hand side and the left hand side of the spectrum. This information can be used along with the detector sensitivity curve and calculated filter vector transmission to decide how much of the spectrum should be utilized to optimize the SNR for a given discrimination problem.

In figure 10, the SM actually improves with the removal of several spectral channels from the left hand side. It appears that it would be helpful to exclude these channels regardless of the edge effect we have just discussed. It is common in multivariate analysis to find that some spectral channels contribute more noise than information, so that 
their exclusion is beneficial to the prediction sought. Of course, the channels to be excluded may also be internal to the spectrum. Genetic algorithms are often employed to arrive at the optimal band set for a given problem. ${ }^{7}$

\section{MULTIPLE FILTER VECTOR APPROACHES}

In difficult discrimination problems a single filter vector based on linear discriminant analysis may be insufficient to provide reliable detection of a target. We are investigating other approaches where several filtered images are collected and then combined to produce a superior result. One possibility is canonical discriminant analysis $^{8}$ which gives an expansion where the first term is the equivalent to equation 1 and additional terms refine the result. Also, we can apply linear discriminant analysis sequentially to separate the classes pairwise and then apply the appropriate Boolean algebra to the series of images to isolate the target of interest. Finally, quadratic discriminant analysis may prove interesting. ${ }^{8}$ Results of these investigations will be presented in a future publication.

One criticism of ISIS is that only the final image is saved while the complete data cube is lost. Would it not be better to retain the complete data cube for later analysis? When possible, of course, the answer is yes. But we envision applying ISIS to tasks that cannot be accomplished with a conventional HSI (see conclusion section and reference 2). Also, we point out that ISIS can, in fact, acquire a complete data cube simply by cycling through the individual filter elements, turning on one at a time. However, this comb-like basis set, which is used in conventional spectroscopy, is very inefficient. More interestingly, ISIS can collect a complete data cube in a multiplexed fashion by cycling through a complete basis set of filter vectors. The relevant transform is then applied to arrive at the spectra. The Hadamard basis set is attractive for this type of spectral acquisition due to the simplicity of its transform. ${ }^{4}$ A complete basis set must have the same the number of filter vectors as there are individual spectral channels in the filter. However, it is not necessary to program ISIS with a complete basis set of filter vectors, unless one wants to record a set of arbitrary spectra. Analysts studying the PCs of a typical remotely sensed image have noted that generally 5-20 PCs are sufficient to represent the scene. We propose that ISIS be programmed to collect images of each filter vector in a truncated basis set. This in effect provides an optical data compression very similar to what others propose to accomplish with onboard computers. Figure 11 shows how a few PCs can be used to reconstruct a representative full spectrum from the image of figure 2 . At the point that the residuals are much smaller than the within-class variance, the fidelity of the reconstructed spectrum is adequate for most applications.

In ref 2 we note that a $\mathrm{PC}$ basis set need not use two ISIS channels for each vector. The first vector is always positive. The subsequent vectors can be made positive by programming them as a linear combination of the first and later PCs. Hence, the number of channels equals the number of PCs desired rather than $2 \mathrm{X}$ the number of PCs desired. Truncated basis sets based on something other than the PC expansion should also be considered. For instance, many have found the end-member expansion to be useful. ${ }^{9}$ It is convenient that each vector in this expansion is non-negative.

\section{FUTURE WORK}

We are now performing experiments with the ISIS, Phase II instrument, which unlike its predecessor is primarily intended for field use. The design is optimized for the full wavelength range of the Si detector from 450 to $1050 \mathrm{~nm}$. The design and performance of the new ISIS will be documented in a future publication. Other designs are contemplated including instruments that will function in the SWIR and LWIR. ${ }^{1}$

There is much work to be done on the multiple vector approaches discussed in section 5. Also, we intend to produce a more detailed comparison of the performance of ISIS to multi- and hyperspectral instruments than currently exists. ${ }^{2,3}$ Atmospheric effects will be included in this comparison. 


\section{CONCLUSIONS}

This paper has shown how to construct filter vectors for an ISIS instrument. Both single and multiple vectors approaches were considered with several suggestions for future work. It was shown that the problem of overfitting and edge effects should be considered when determining the best possible filter vectors.

ISIS is a unique concept that provides the possibility of compressing the data in a large data cube by optical means. This is important to improve the SNR, lower the demands on the communication systems, and provide real time target identification. In the single vector mode ISIS produces directly the dot product of the image with a discriminant vector chosen by the user. This gives a gray scale, or thresholded binary image of the target versus the background. In the multiple vector mode ISIS can improve the reliability of the target detection. Also, it can acquire a truncated data cube with only a few spectral images that have been chosen to retain efficiently the information essential to the problem at hand. If desired, one may transform the truncated data cube to a traditional full spectrum result.

Conventional HSI instruments pay a price for high spectral resolution. In satellite applications, the SNR may be unacceptably low if one also requires broad area coverage (i.e., speed), or high spatial resolution. ${ }^{2}$ We do not envision ISIS as a general replacement for HSI, but rather an alternative in applications where at least one of these later two requirements is critical.

\section{ACKNOWLEDGMENTS}

Sandia is a multiprogram laboratory operated by Sandia Corporation, a Lockheed Martin Company, for the United States department of Energy under contract DE-ACO4-94AL85000.

\section{REFERENCES}

1. W.C. Sweatt, C.A. Boye, S.M Gentry, M.R. Descore, B.R. Stallard, and C.L. Grotbeck, "ISIS: an informationefficient hyperspectral imaging sensor," Proc. SPIE, 3438, pp. **, 1998.

2. B.R. Stallard, S.M. Gentry, W.C. Sweatt, S.E. Motomatsu, and C.A. Boye, Principles and Satellite Applications of the Information-Efficient Spectral Imaging Sensor, (Sandia National Laboratories, SAND97-1493, 1997). Distribution is currently limited to US government agencies due to patent caution. Authors have requested status change to unlimited distribution which is likely to be approved in Fall 1998.

3. S.M. Gentry and B.R. Stallard, Theoretical Analysis of the Sensitivity and Speed Improvement of ISIS Over a Comparable Traditional Hysperspectral Imager, (Sandia National Laboratories, SAND98-****, 1998).

4. M. Harwitt and N.J.A. Sloane, Hadamard Transform Optics, (Academic Press, New York, 1979).

5. K. Fukunaga, Introduction to Statistical Pattern Recognition, (Academic Press, New York, 1990).

6. T.J. Hastie, A. Buja, and R. Tibshirani, "Penalized Discriminant Analysis," Annals of Statistics, 1995.

7. E.M. Bassett and S.S. Shen, "Information Theory-Based Band Selection for Multipsectral Systems," Proc. SPIE, 3118, pp. 28-35, 1997.

8. R.A. Johnson and D.W. Wichern, Applied Multivariate Statistical Analysis, (Prentice Hall, Englewood Cliffs, 1992).

9. F.A. Kruse, A.B. Lefkoff, J.W. Boardman, K.B. Heidebrecht, A.T. Shapiro, P.J. Barloon, and A.F.H. Goetz, "The Spectral Image Processing System (SIPS): Interactive Visualization and Analysis of Imaging Spectrometer Data," Remote Sensing of Environment, 44, pp. 145-163, 1993. 


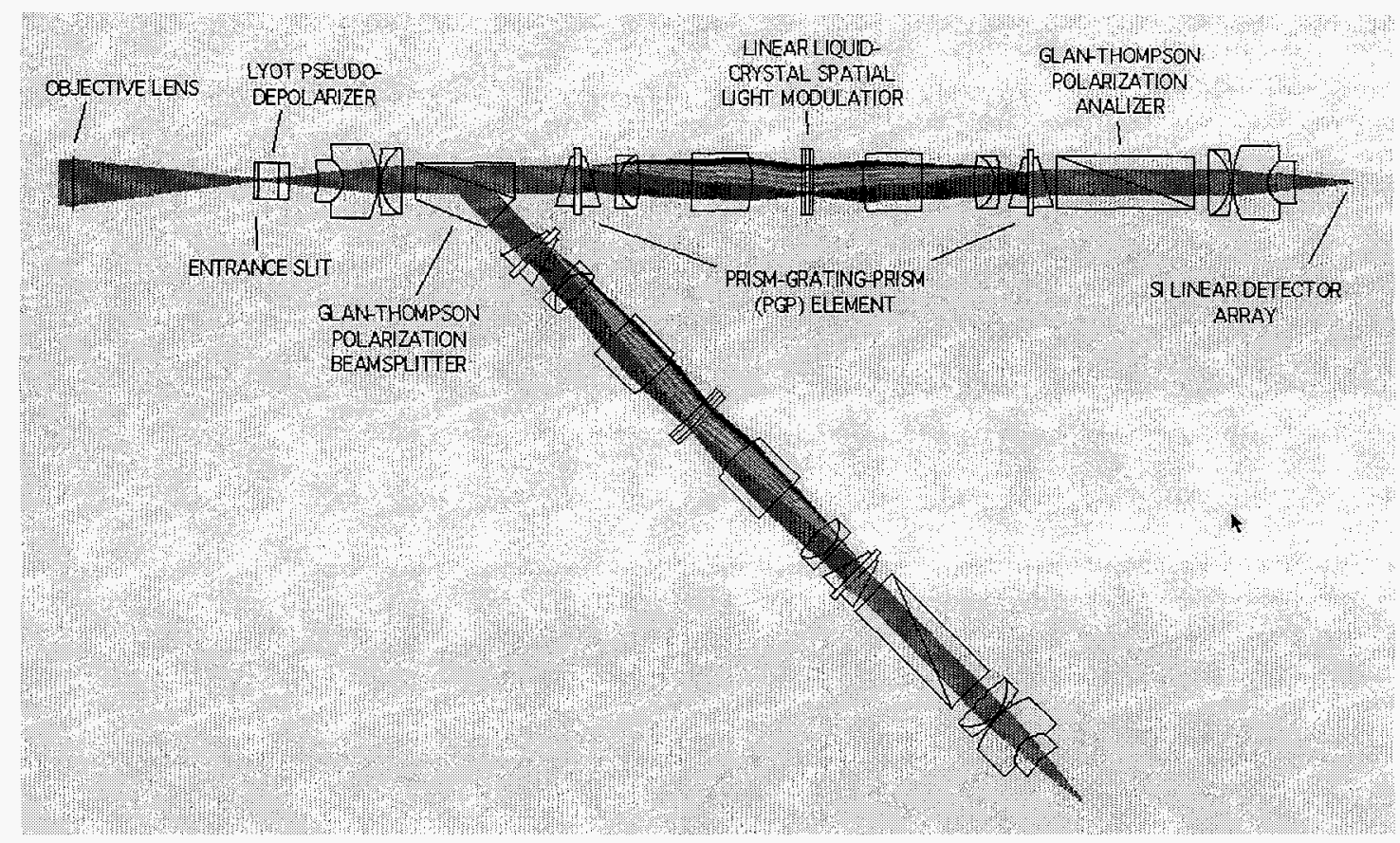

Figure 1. Schematic diagram of the type of ISIS instruments constructed at Sandia National Laboratories.
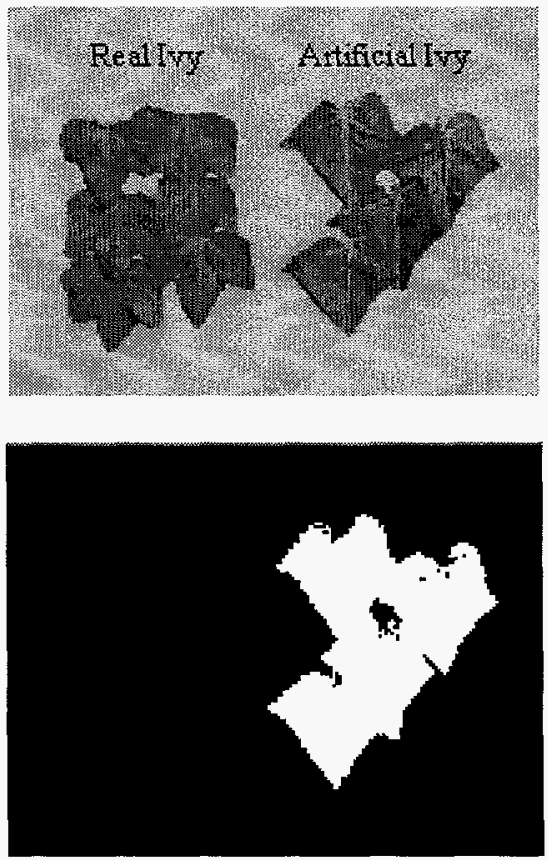

Figure 2. A photograph (above) and an ISIS image (below) of a few leaves of real ivy and artificial ivy. A threshold was applied to the ISIS image so that the pixels are either 0 or 1 . The ISIS image can also be displayed as a 128 level gray scale. 


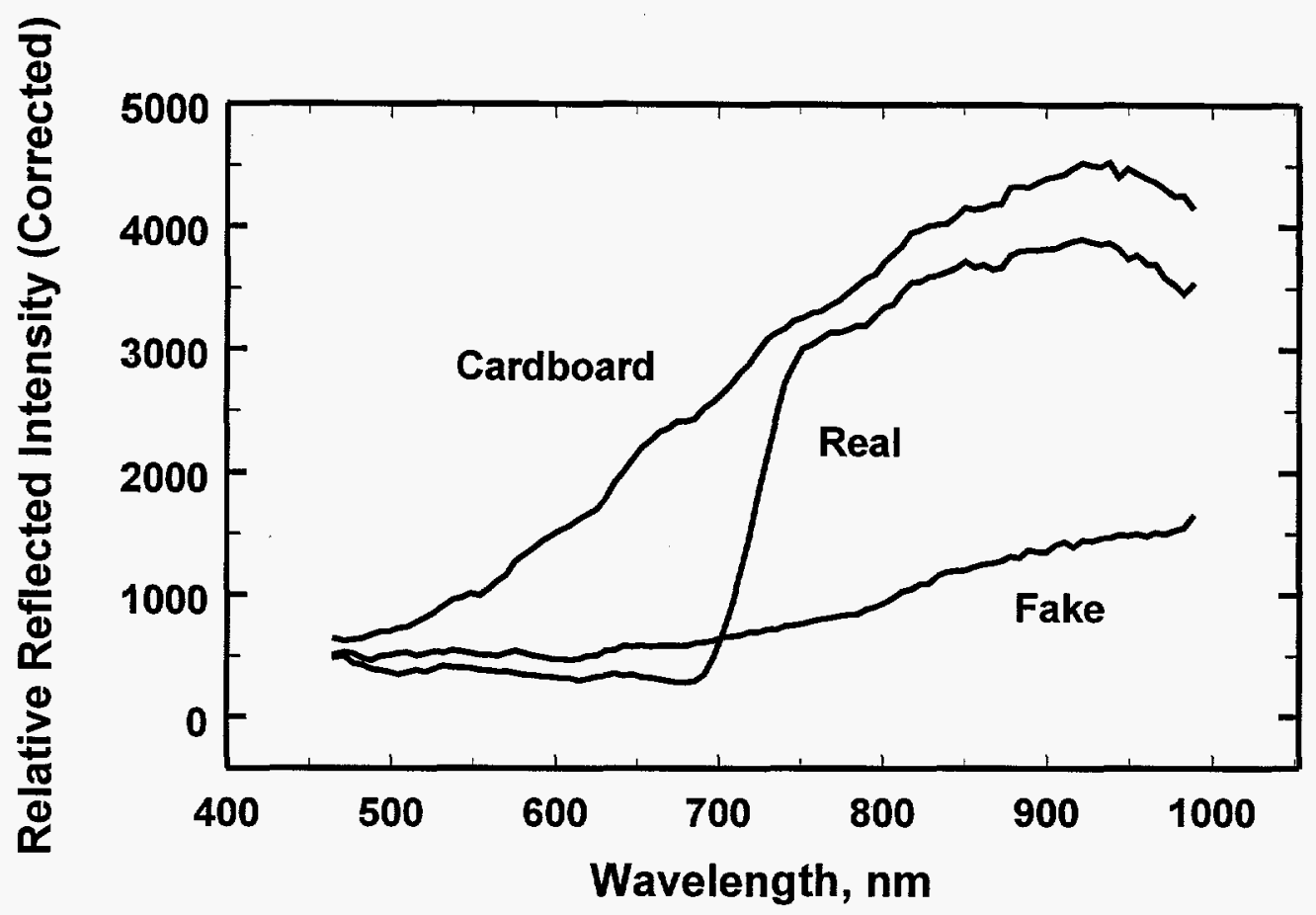

Figure 3. Mean spectra for the three materials in the image in figure 2.

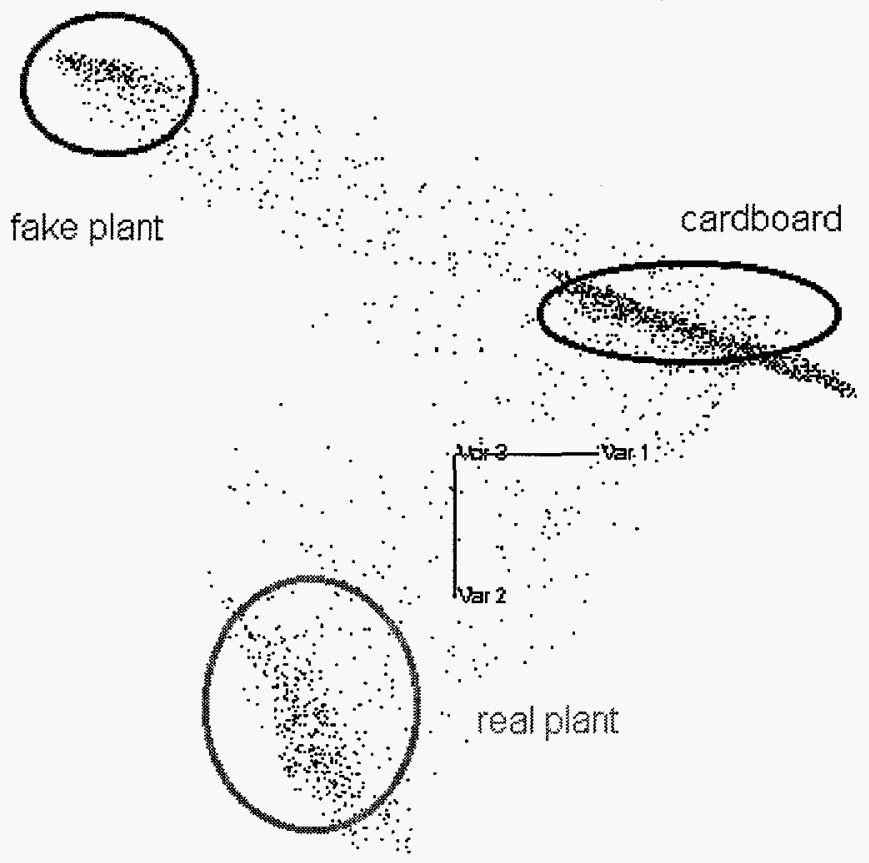

Figure 4. A scatter plot of the pixels in the hyperspectral image (not shown) of the objects in figure 2. The axes labeled Var 1, Var 2, and Var 3 are the first, second, and third principle components, respectively. Note that Var 3 is nearly perpendicular to the page. 


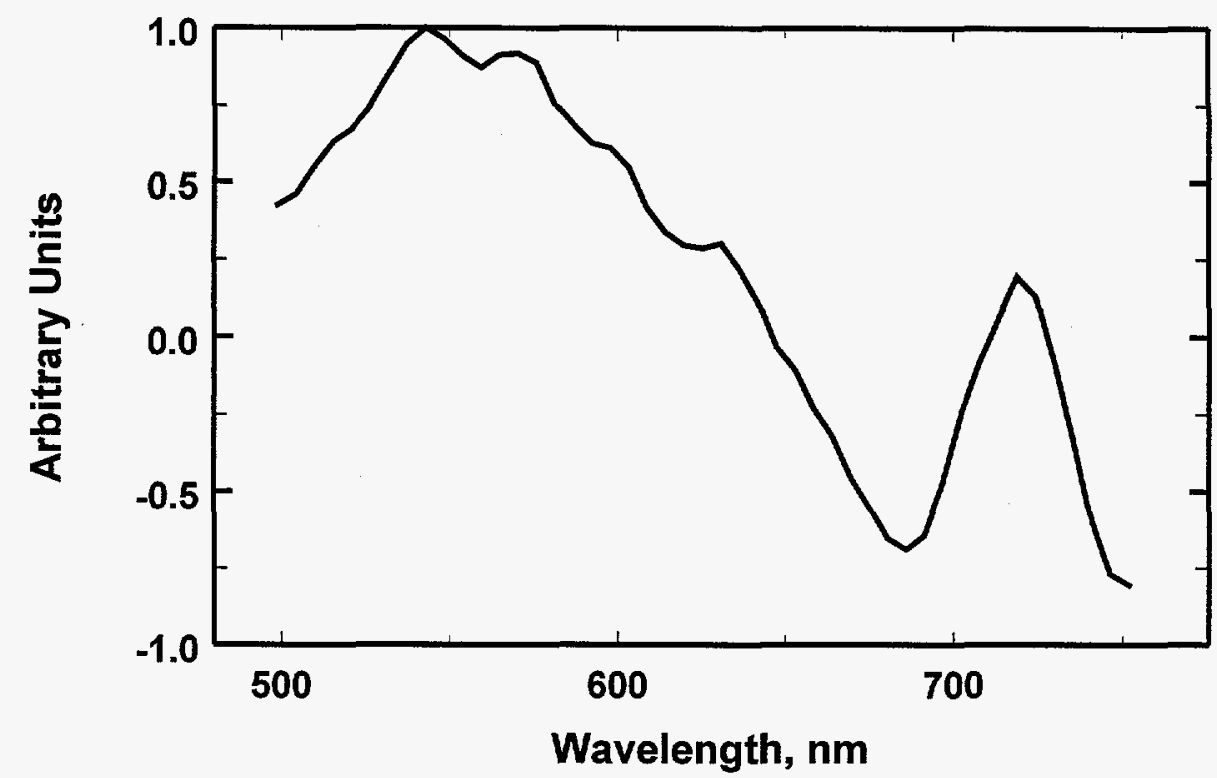

Figure 5. The filter vector used in to obtain the ISIS image in figure 2. It is the linear discriminant vector as explained in the text..

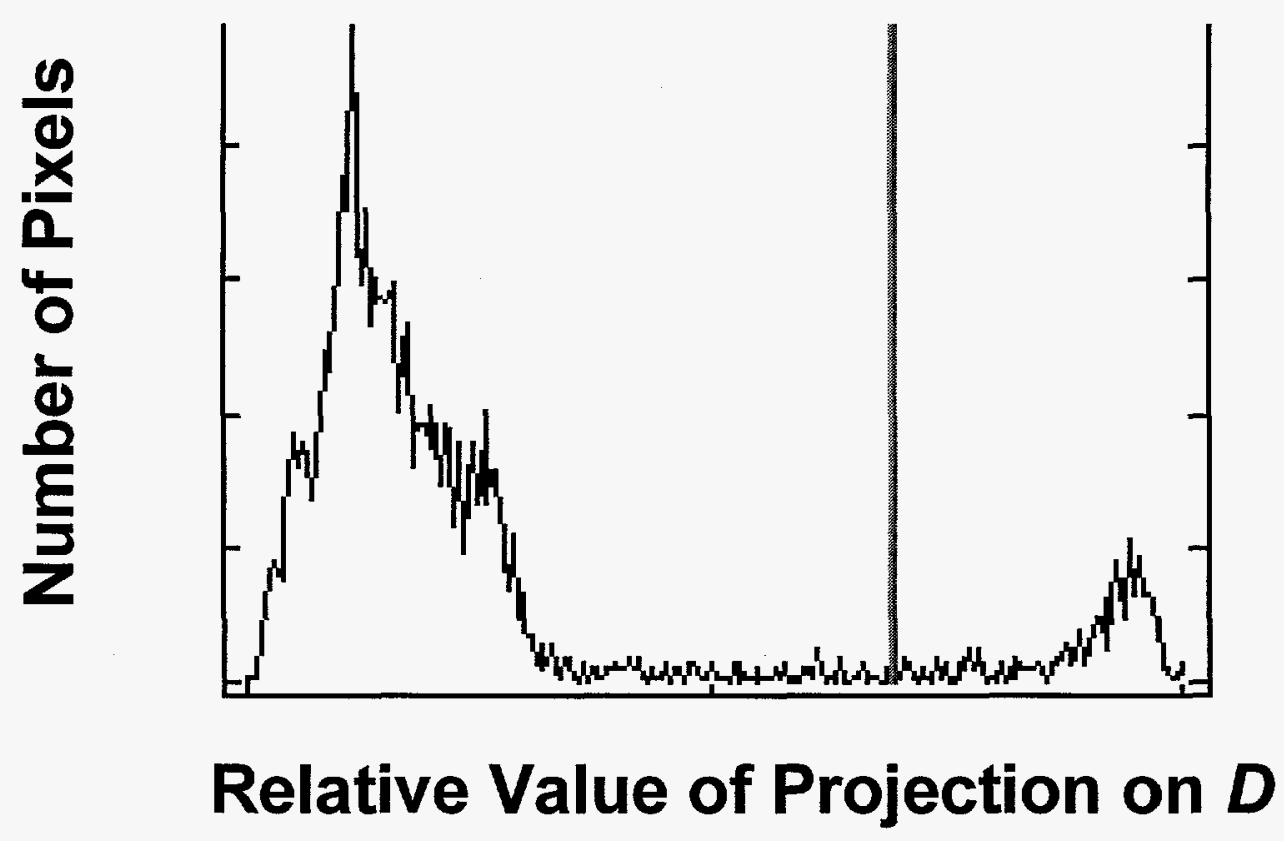

Figure 6. A histogram of the scores for the pixels in the ISIS image of figure 2 (before thresholding). The vertical line is the threshold used to obtain the binary image shown in figure 2 . 

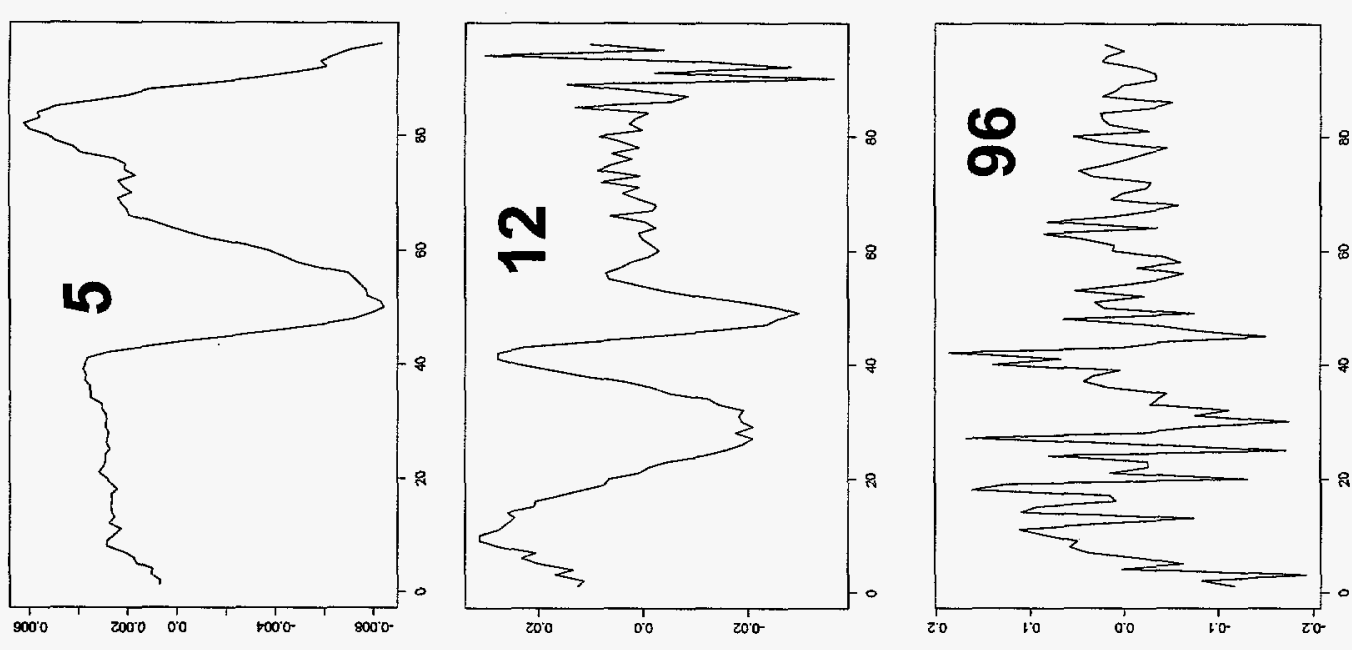

定
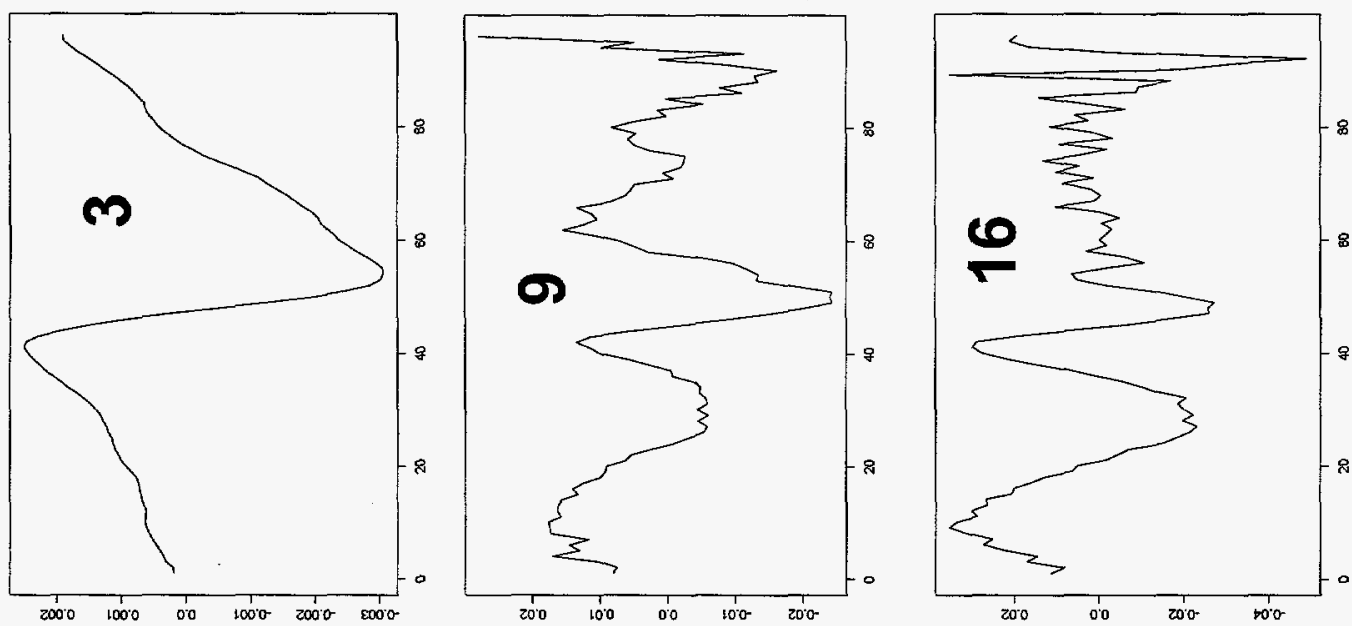

.
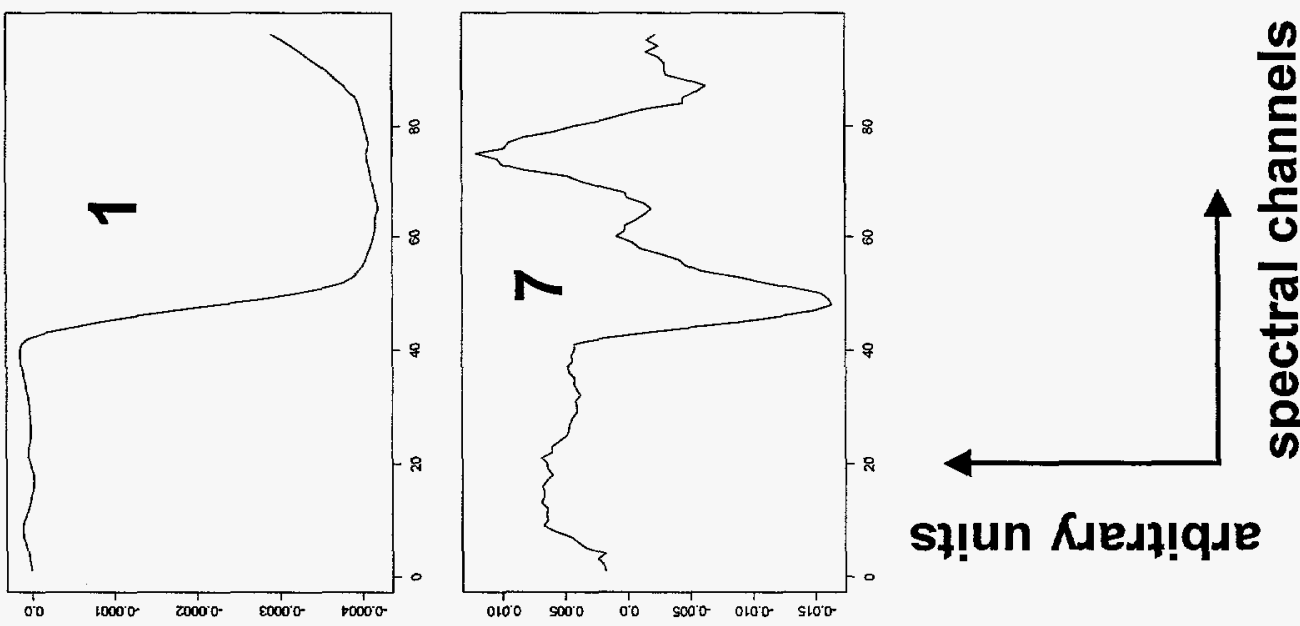

品 


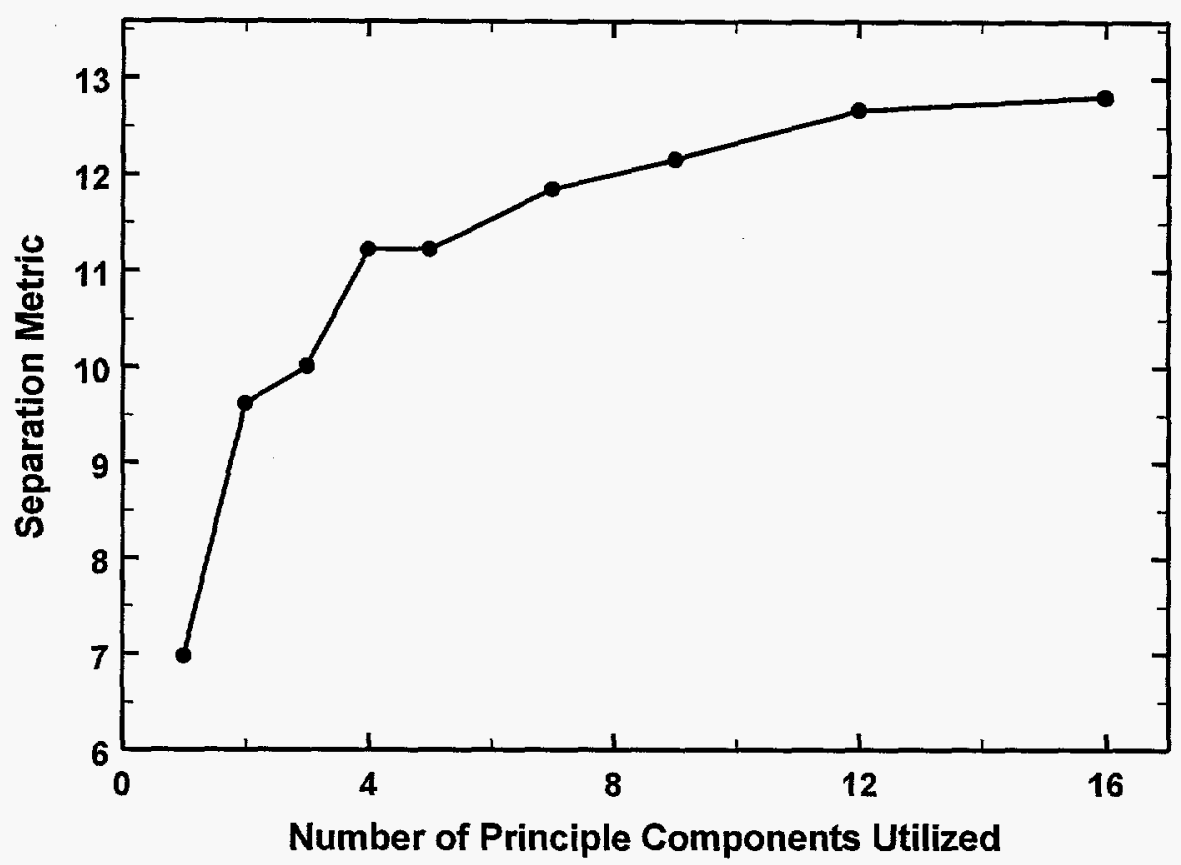

Figure 8. A plot of the Separation Metric as a function of the number of PCs used in the construction of the linear discriminant vector.
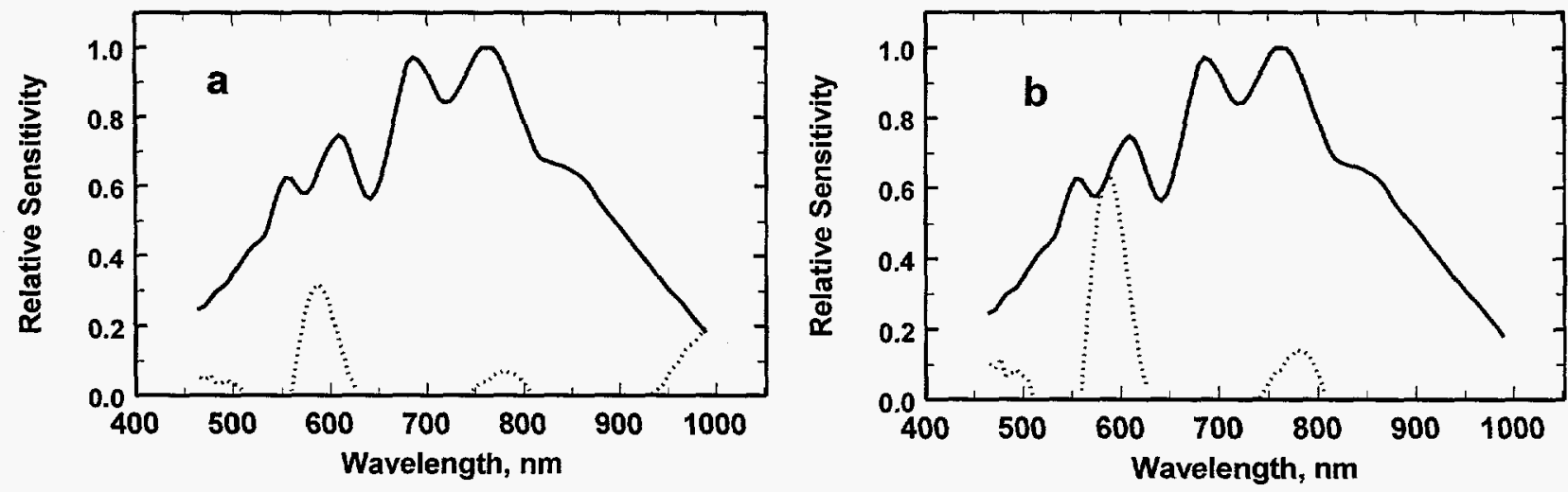

Figure 9. Plots showing the interplay of the filter vector (dotted line) with the sensitivity of the detector (solid line). a) when the complete filter vector extending from 450 to $1000 \mathrm{~nm}$ is used. b) when only the spectral range from 450 to $940 \mathrm{~nm}$ is utilized. The transmission of the filter vector in $\mathrm{b}$ is higher. 


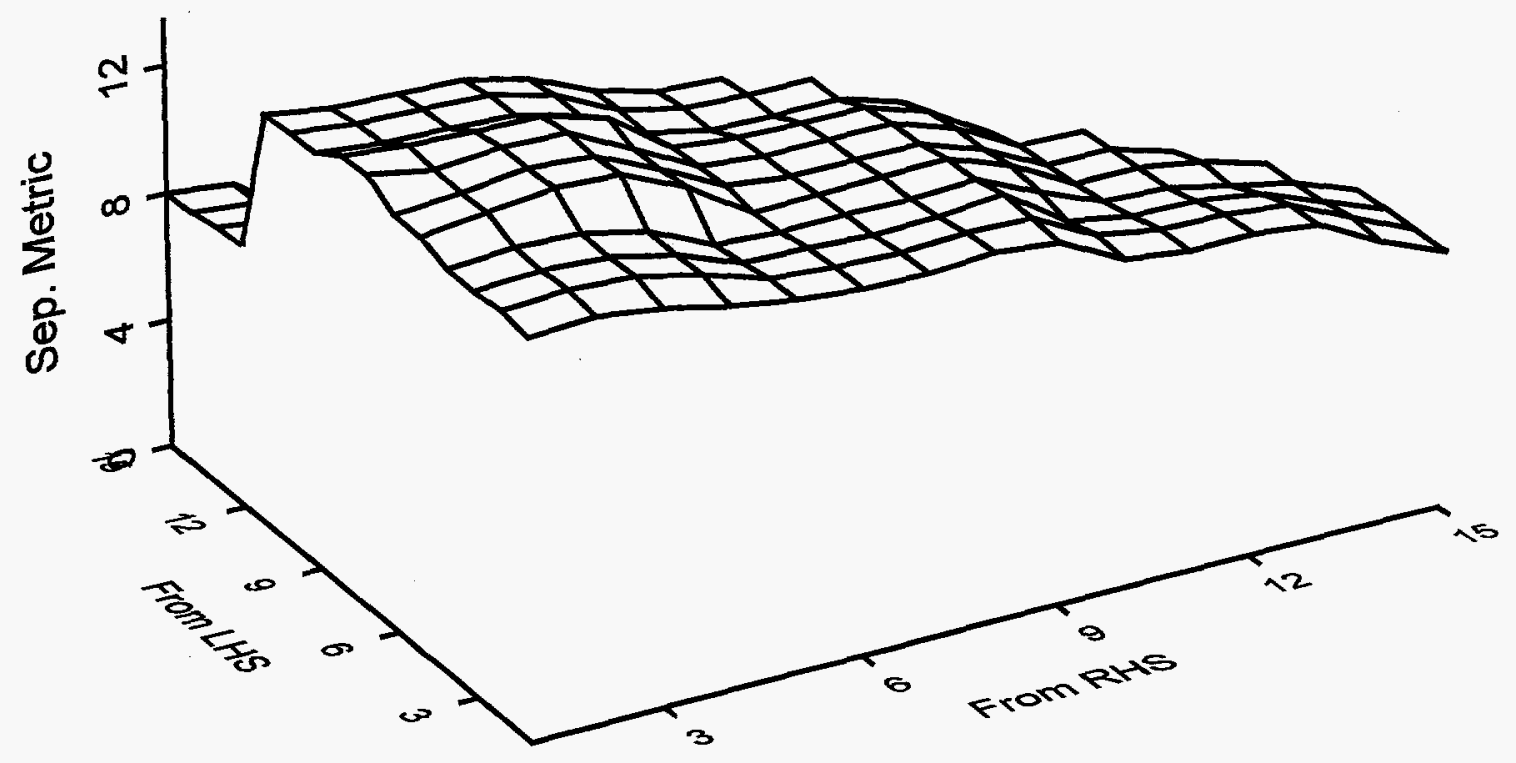

Figure 10. A plot of the separation metric as a function of excluding spectral channels (each about $5 \mathrm{~nm}$ in width) from both the LHS and RHS of the spectra in figure 3.
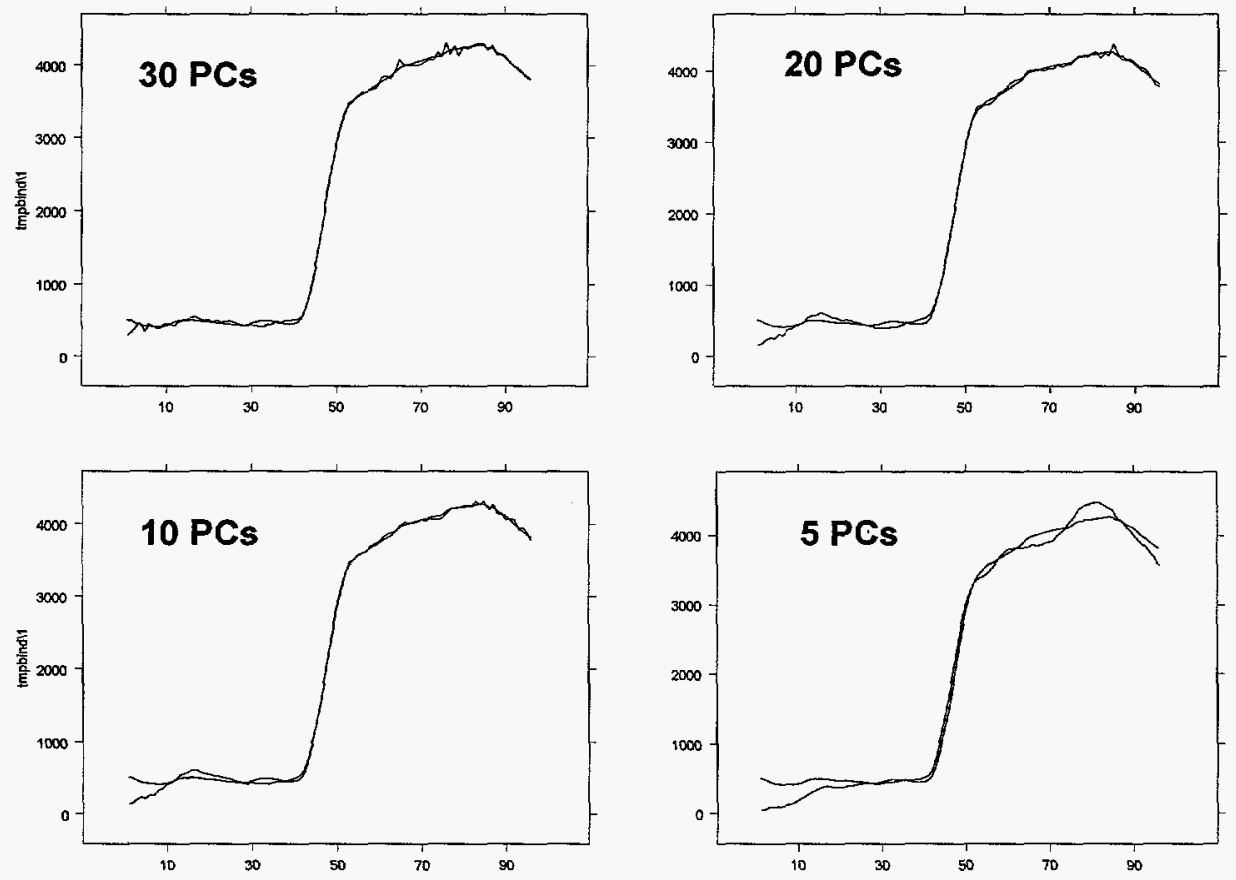

Figure 11. A comparison of reconstructed spectra plotted over an actual spectrum of the real plant from figure 3 The number of PCs used are indicated in each subfigure. The relatively smooth curve that is constant among the subfigures is the true spectrum. 